\title{
ON THE STRUCTURE OF THE MAGNETIC FIELD OF SUNSPOTS
}

\author{
E. I. Mogilevsky, L. B. Demkina, B. A. Ioshpa, and V. N. Obridko \\ (Institute of Terrestrial Magnetism, Ionosphere and Radio-Wave Propagation of the \\ USSR Academy of Sciences, Moscow')
}

\begin{abstract}
The model of the magnetic field of sunspots, taking account of fine structure of magnetic field in solar plasma, is considered. Small-scale subgranules with their own field form magnetic filaments in the external current-free field. The filaments are vertical in the umbra, while in the penumbra they run along the surface with sharp bends. In a number of spot umbra the relation between Doppler velocity and the field is established on polarized spectrograms. The $\pi$-component splitting in umbra is interpreted as a result of a weak background magnetic-field existence together with a large field of magnetic filaments. Spectrographic definition of the magnetic field in spot umbra is accomplished on the effect of magnetic-lines intensification and directly on spectrograms of lowexcitation ( $\mathrm{Fe}$ I, $\mathrm{Ti}_{\mathrm{I}}$ ) and high-excitation ( $\mathrm{Fe}$ II) lines. Magnetic field measured in low-excitation lines exceeds twice the field value obtained in high-excitation lines. This result has been considered in the light of the proposed model of sunspot field.
\end{abstract}

By studying the magnetic field of a sunspot one can obtain the most comprehensive information on the small-scale structure of the solar magnetoplasma. This is primarily due to the fact that the photographic method is the most efficient in measuring sunspot fields, since it yields a high resolution and good guiding. On the other hand, investigation of the magnetic-field distribution separately in the umbra and penumbra of a sunspot makes it possible to study the structural elements in two projections, as it were, i.e. from above in the umbra, where the field is predominantly vertical, and from the side in the penumbra, where the field is near horizontal. Finally, the sunspot field develops rather rapidly and evidently remains largely unaffected by the magnetic fields of neighbouring activity phenomena. A working model of a magnetic field of a sunspot, which takes into account the small-scale structure of the magnetoplasma and some spectral observations shedding light upon the fine structure of the magnetic field of a sunspot will be considered below.

The model of the magnetoplasma structure considered by Mogilevsky and Shelting (1968) implies the existence of small-scale $\left(\sim 10^{7} \mathrm{~cm}\right)$ elements (so-called sub-granules) with a closed magnetic field surrounded by a quasi-homogeneous current-free magnetic field $H=H_{0}+\sum_{i} H_{i}$, where $H_{0}$ is the homogeneous magnetic field and $\sum H_{i}$ is the total field (at the given point) of the entire ensemble of subgranules.

Due to the interaction between their magnetic moments, sub-granules tend to line

Kiepenheuer (ed.). Structure and Development of Solar Active Regions, 215 229. C. I.A.U. 
up in long chains (magnetic filaments) whose space distribution determines the observed large-scale field of the spot. In the umbra we should observe a receding ensemble of vertical magnetic filaments which can group together into convection cells owing to convective sub-photospheric motions (which obviously take the form of oscillatory convection). The first appearance of a pore corresponds to the extending of a magnetic filament cell with a mean intensity of $\gtrsim 1200-1500$ oe. Neglecting the problem of the generation of sub-granules with a force-free magnetic field*, one may assume that the subsequent growth of the pore is a process of the successive extending of vertical chains of sub-granules grouped together into convection cells. It follows from observations (Vasilieva, 1963) that this process of the initial growth of the spot is preceded by the egress of the gas-dynamic shock-wave to the photosphere.** The wave, propagating partly upwards, carries the initial weak magnetic field of the future spot out into the chromosphere. Extending vertical magnetic tubes can extend into the chromosphere and corona through the initial field, because the diffusion of sub-granules over the gradient field is much more rapid (by a factor of the order of the Reynolds' magnetic number) than ordinary magneticfield diffusion (Syrovatsky and Zhugzhda, 1967).

The umbra will continue growing until part of the outer magnetic tubes begin to deviate from the vertical, due to refraction when the field propagates into the chromosphere where the conductivity increases. This deviation will result in the bending of the outer filaments and later in their return into the photosphere (the outer boundary of the penumbra). These magnetic filaments, creeping through the photosphere, form a relatively thin $\left(\simeq 5 \times 10^{7} \mathrm{~cm}\right)$ translucent magnetic surface, the penumbra. The qualitative representation of this scheme is given in Figure 1. In this working model, the umbra of the spot is an inhomogeneous (due to convective perturbations) band consisting of sub-granule filaments whose vertical field may recede into the region of the chromosphere and corona and later close upon the umbra (or umbras) of other spots of the group, while the penumbra is a relatively thin layer of magnetic tubes detached from the umbra which yield a near horizontal field and close either under the photosphere or upon the dark intergranular areas of the photosphere. The essential point in this 'mushroom-like' schematic model of the spot is that the formation of the penumbra due to the bending of the magnetic tubes should be accompanied by substantial changes in the magnetic flux and by the generation of a ring current in the surface layer of the photosphere with a finite conductivity. The field of this toroidal current, located under the penumbra around the umbra, determines the stability of the sunspot umbra at the photosphere level. The ring-current energy, which is equal to or less than the energy of the penumbra field, may be estimated by calculating the

* That the field in sub-granules tends to become force-free follows from the condition of magneticenergy extremum in the sub-granule.

** This is accompanied by the appearance of a bright-point floccule and then of a small ringlike one. 


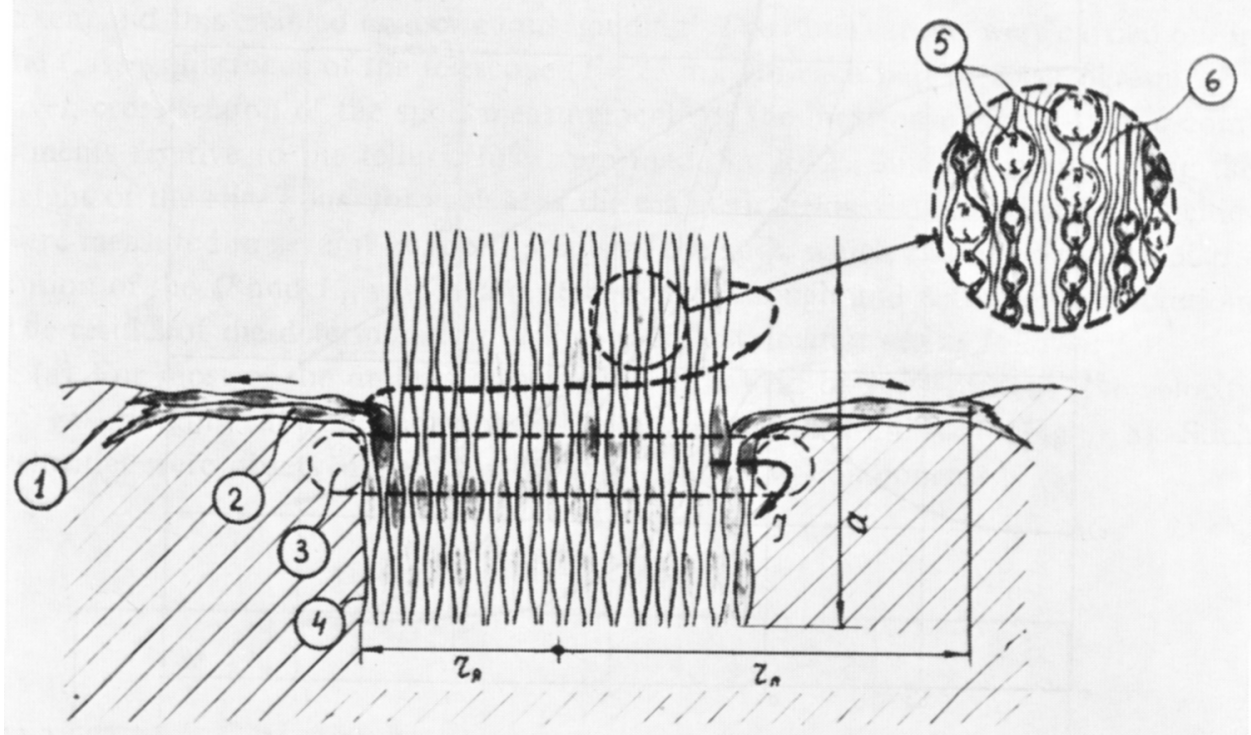

FIG. 1. Structural scheme of the magnetic field of a sunspot: $1=$ photosphere; $2=$ penumbra field; $3=$ induced ring current restraining the umbra field; $4=$ umbra field; $5=$ sub-granules in various convection cells; $6=$ current-free background magnetic field.

field of the ring at the characteristic values given in Figure 2. It was assumed that the mean field value in the penumbra was $\$ 0.25 H_{\max }$, and therefore the ring was located at a depth of about $\mathbf{0 . 2}$ umbra radius (Kolpakov, 1966). The addition of the 'table-shaped' umbra field, the penumbra field and that of the 'holding' ring opposite in sign yields a sunspot field similar to the observed distribution (Figure 2). Here we only consider the magnetic-field intensity distribution. It is known, however (Severny, 1964) that the observed distribution of the sunspot-field vector is not approximated by the dipole field, nor is it a potential one, because the observations point to the existence of an azimuthal component. Restricting ourselves to the general structural scheme of the spot and without going into the nature of the field-vector distribution, we will note that the set of vertical magnetic tubes of the umbra should acquire the general azimuthal component due to the helical instability of the plasma with the field (Kadomtzev, 1962). In this case the general magnetic field of the umbra (especially in the chromosphere) will also be force-free (Mogilevsky and Shelting, 1966). With the growth of the umbra area ('surfacing' or 'sinking' of identical-type magnetic 


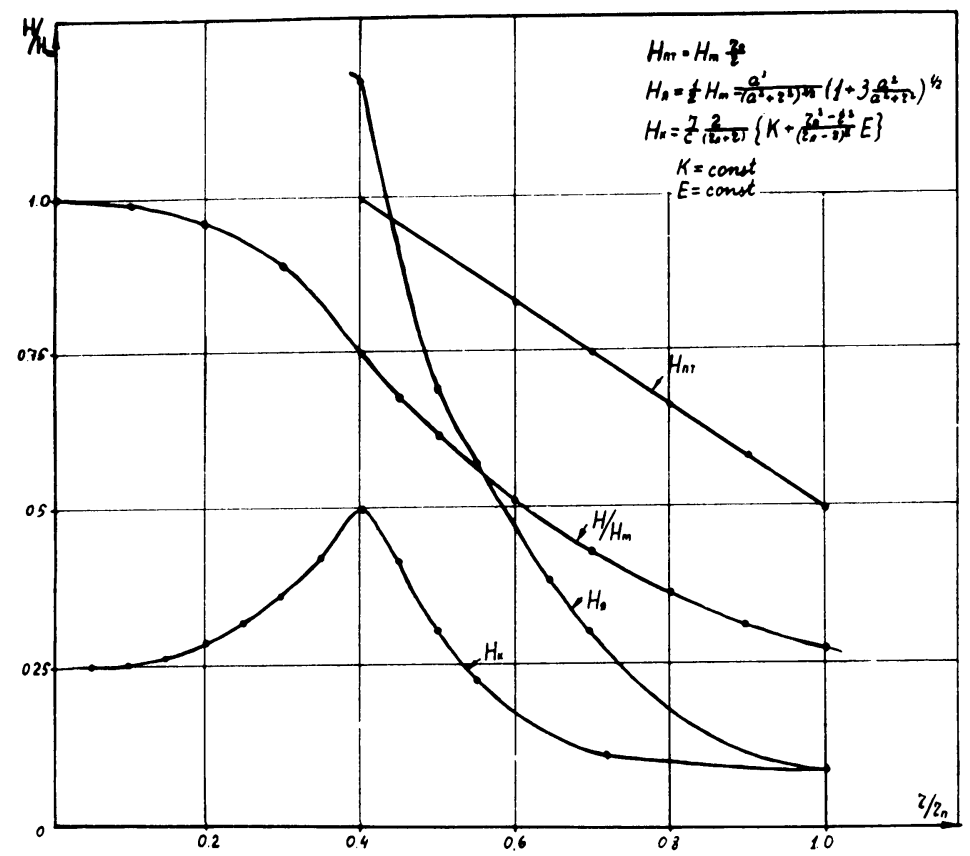

Fig. 2. Distribution of magnetic field $H$ in sunspot: $H_{u}=$ umbra field, $H_{n m}=$ penumbra field, $H_{k}=$ ring-current field, $H_{\max }=$ maximum intensity in sunspot umbra.

filaments of the sub-granules) the specific tension (stress per unit length) of the ring is retained, which is in accord with its stability. The dissipation time of the ring current, primarily due to Joule losses (taking into account the conductivity according to Kuklin, 1966, in partly ionized sub-photospheric layers) will be of the order of $5 \times 10^{6}-10^{7} \mathrm{sec}$, which corresponds to the lifetime of the spot. The obvious implication of this sunspot model is the possibility of a considerable penetration of the umbra field into the chromosphere and the small height of the penumbra. The relatively small $\left(\$ 10^{8} \mathrm{~cm}\right)$ thickness of the penumbra naturally explains its sharply defined boundaries (photosphere-penumbra, penumbra-umbra), whose contrast does not vary with the shifting of the spot from the centre towards the limb. The filamentary structure of the penumbra aligned along the field and the near-round granules in the umbra, the nature of the gas motion within the umbra and penumbra, and finally the possibility of explaining the spot evolution, as well as some morphological features of complex (multi-nucleus) spots within the framework of this mode speak in favour of the working model under review.

To obtain additional information on the structure of the sunspot magnetic field, detailed photographic measurements of the magnetic-field distribution in a great number (about 50 ) of large ( $\mathrm{Sp} \gtrsim 250$ units), predominantly quiet spots were carried out with the tower solar telescope IZMIRAN in 1966-67 (Zhulin et al., 1966). For 
this purpose paired polarization spectrograms near line $\mathrm{Fe}$ I $\lambda 6302.508 \AA$ were obtained by traversing the spot $15-25$ times with the spectral slit. Simultaneously with taking the spectrograms, a series of pictures of the spot on the spectrograph slit were taken, and this enabled unambiguous 'guiding'. The observations were carried out in the Cassegrain focus of the telescope $(F=27 \mathrm{~m})$. On each pair of spectrograms of a given cross-section of the spot, measurements of the location of the $\pi$ - and $\sigma$-components relative to the telluric line were made in 20-25 different points along the height of the slit. Thus, for each spot the magnetic fields and line-of-sight velocities were measured in several hundred points of the spot, which characterized the distribution of the $H$ and $V_{\mathrm{D}}$ values comprehensively enough and with a high resolution. The results of the determination of line-of-sight velocities are as follows:

(a) For most of the umbras measured, with a field of 2000-3500 oe, the velocity of gas 'sinking' at the photosphere level was about $1 \mathrm{~km} / \mathrm{sec}^{*}$ (Figure 3). Such velocities were observed both for the $\sigma(0)$ - and $\pi(\nabla)$-components.

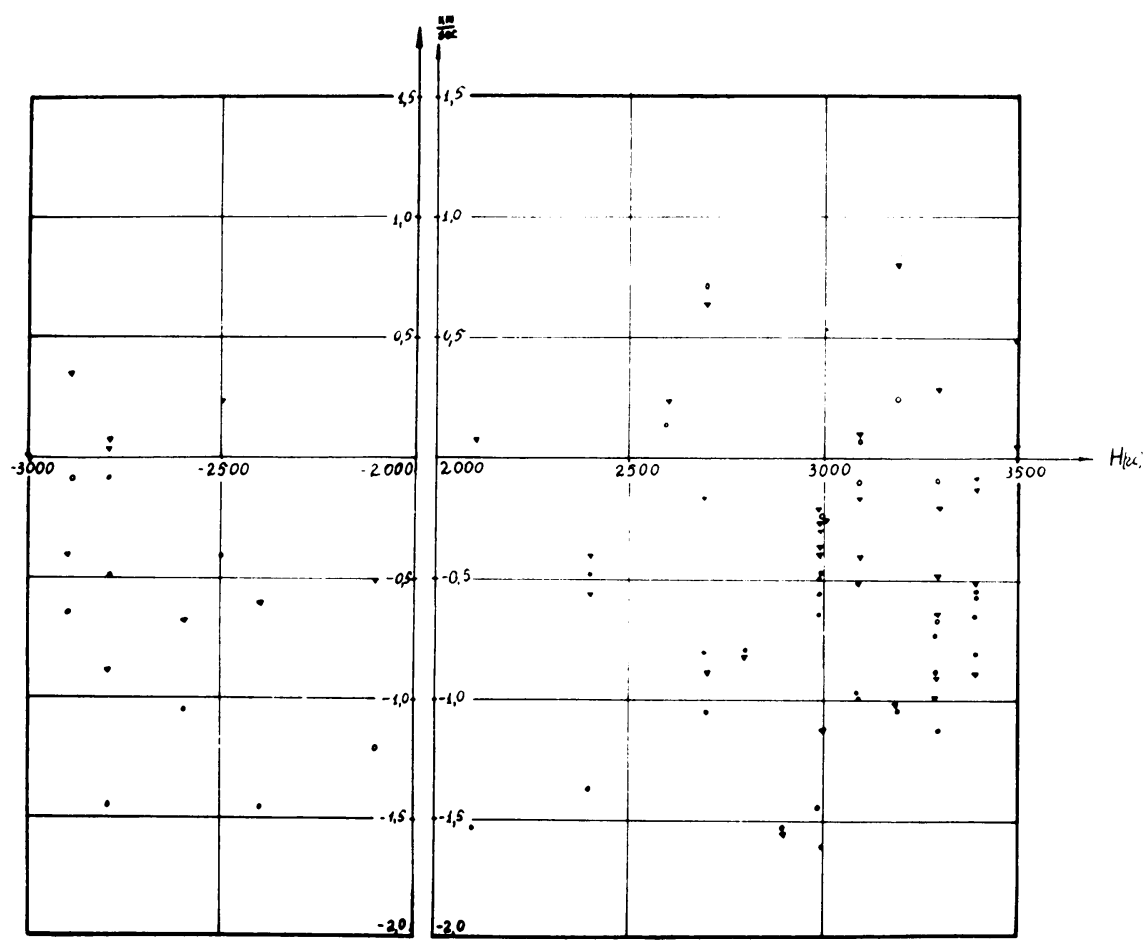

FIG. 3. Dependence of line-of-sight velocities $V_{D}$ in sunspots defined from $\sigma(0)$ - and $\pi(\nabla)$-components, on magnetic field $H$ (taking account of polarity $+S,-N$ ).

* The sign of the velocity (descent or ascent) may depend on the phase of sunspot development. Since we used only spots of large area, we were able to observe mainly the descent of the gas which is characteristic of the period of the post-maximum development of the group. 
(b) The feasibility of defining the line-of-sight velocities by the $\sigma$ - and $\pi$-components is illustrated in Figure 4. A linear relationship of the type $V_{\sigma}=V_{\pi}-0.26 \mathrm{~km} / \mathrm{sec}$ was found between these velocities $\left(V_{\sigma}\right.$ and $\left.V_{\pi}\right)$. The ray shift of the lines indicates that, at least in the umbra, the role of the scattered light which would yield unshifted lines is not great.

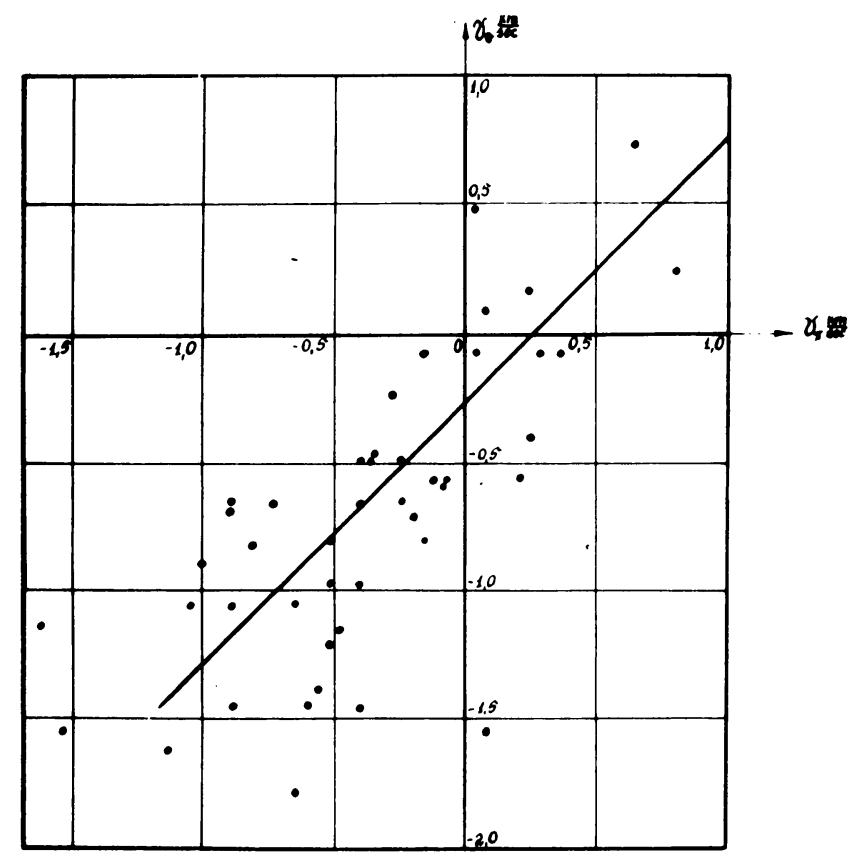

Fig. 4. Comparison of line-of-sight velocities in sunspot umbra obtained by measurements in $\pi\left(V_{.}\right)$ and $\sigma\left(V_{\sigma}\right)$-components.

(c) The existence of relatively high vertical velocities in the umbra, obtained by photographing, was confirmed by photoelectric measurements of the same spot with a magnetograph (for a large spot of July 19, 1966, which was located near the central meridian of the Sun).

Another important result promoting the understanding of the sunspot-field structure and obtained from the same materials, was the effect of the $\pi$-component splitting into two sub-components displaced relative to the centre of the line (Figure 5). A similar effect with a complete splitting of the line in the umbra was observed by a number of authors (for example, Severny, 1959; Bumba, 1962; Chistjakov, 1967; and others). It is also revealed by examining photocopies of spectrograms of large spots from the Monte Mario Observatory. This effect cannot be attributed to instrumental polarization, it remains at a considerable turn of the spectrograph grating, for instance 


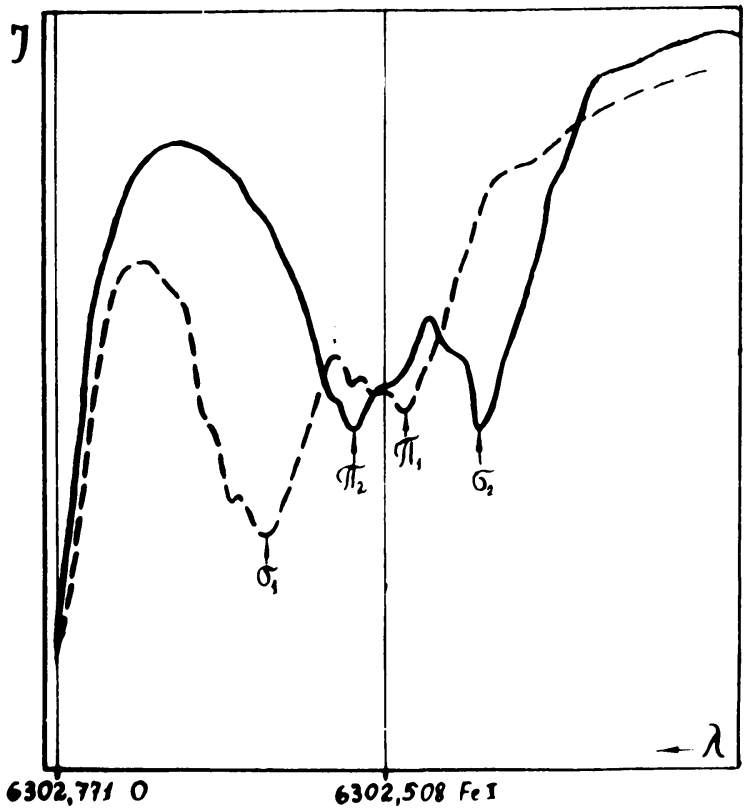

FIG. 5. Example of registrogram of polarization spectra in the region of line $\mathrm{Fe}$ I $\lambda 6302.5 A$ : $l=$ polarization in spectrum $\bigcirc, 2=$ polarization $C$.

upon transfer from the fourth right spectral order to the second left one (Chistjakov, 1967). Consideration of this effect of the $\pi$-component splitting in the umbras for 34 Southern-polarity and 10 Northern-polarity spots (observations of 1966-67) leads to the following conclusions:

(a) The value of splitting, obtained from a great number of points, is $0.008-0.020 \AA$, thus considerably exceeding the possible measurement error (r.m.s. error is $0.003 \AA$ $\pm 10 \%$ ).

(b) The displacement of the split $\pi$-component relative to the centre of the line in each spectrum is independent of the field sign and appears to be opposite to the displacement of the $\sigma$-component (Figure 6 ). The value of the $\pi$-component splitting into two sub-components was found to be about one order less than the distance between the $\sigma$-components.

As was shown in Rachkovsky's (1962) paper, such an effect of the $\pi$-component splitting could have been observed due to the rotation of the polarization plane in an inhomogeneous magnetic field. One must assume that the sense of the rotation of the polarization plane and, accordingly, the sign of the rotation of the field's tangential component, reverse with depth simultaneously with the change of the sunspot-field polarity. Besides, such an effect should be equal in value and sign for all umbra points, which often belong to a markedly inhomogeneous field of the umbra. At the 
same time, Severny's $(1964,1965)$ observations revealed a substantial inhomogeneity of the rotation effect of the polarization plane.

Another interpretation of this effect proceeding from more general possible conceptions of the structure of the magnetoplasma field (Mogilevsky and Shelting, 1968) is that the $\pi$-component splitting should be attributed to the real background magnetic field, whose sign should be opposite to that of the sub-granule field and whose intensity is much lower (depending on the geometry of the sub-granules, their sizes, etc.) than the maximum intensity of the field inside the sub-granules. Here, some relationship should exist between the field of the sub-granules $\left(H_{\sigma}\right)$ and the background $\left(H_{\pi}\right)$ magnetic fields, in accordance with observations (Figure 6), whereas in

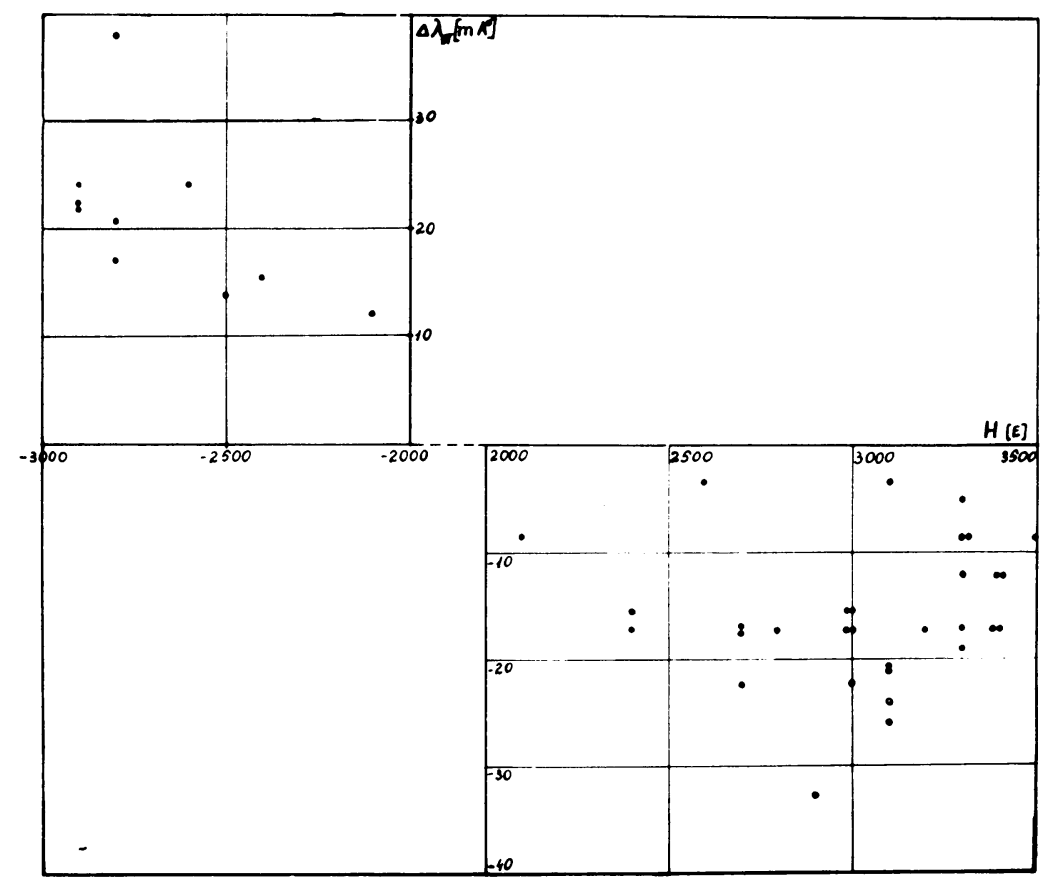

FIG. 6. Splitting of $\pi$-component of line Fe I 6302.5 A as a function of sunspot umbra field $H$.

the general case the rotation of the polarization plane is independent of the value and direction of the field.

One could try to clear up the question of the reality of the background field by considering the effect of the $\pi$-component splitting for the umbras of spots located at different distances from the central meridian of the Sun. Unlike all other possible effects, the value of the background field should depend only slightly on the longitude of the spot. Measurements in 44 spots (without distinction of polarities) were used. 
The result of the statistical analysis is presented in Figure 7. The curve obtained by the least-squares method has the form $\Delta \lambda_{\pi}=0.64 \cos \theta+0.03 \sin \theta+0.44$. In spite of the considerable scatter of the points one can see that, along with $\cos \theta$ terms, nearly half of the mean value of splitting is independent of the longitude.

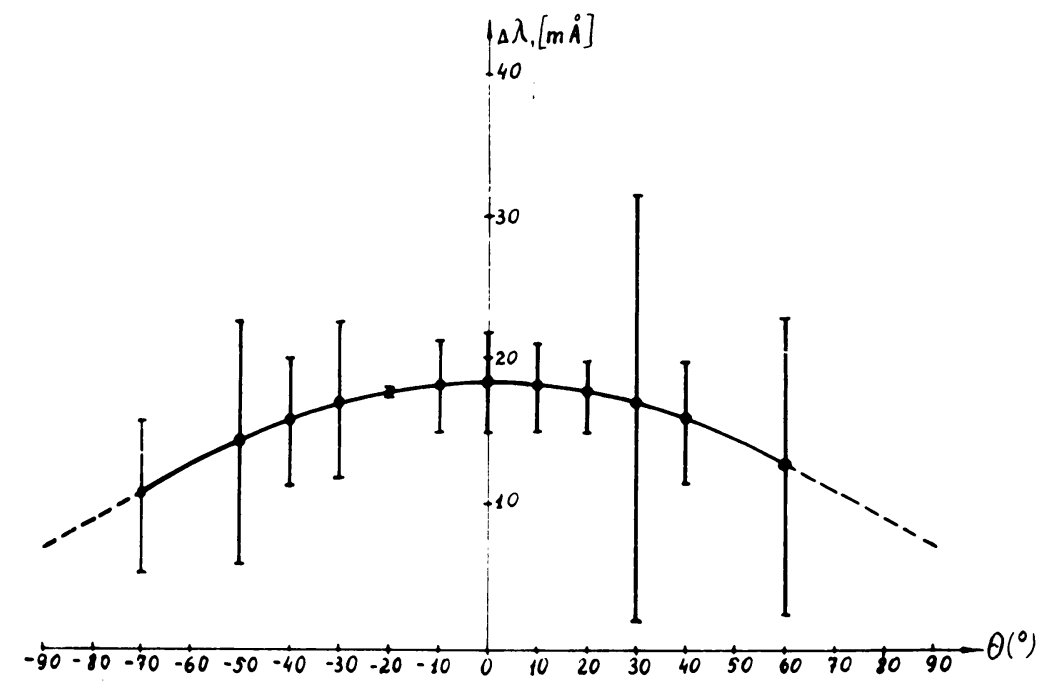

FIG. 7. Dependence of $\pi$-component $\left(\Delta \lambda_{\pi}\right)$ splitting in line Fe $\mathrm{I} \lambda 6302.5$ A on location of spots in the disc.

Note that the detection of such a relatively weak background field is possible only by the photographic method because of the averaging of these fields by the magnetograph.* Since the effect of the $\pi$-component splitting is of great importance for defining the sunspot-field structure, it is necessary to continue detailed investigation such as measurements within various lines and at various heights, the use of a photoelectric magnetograph for determining the variation of polarization along the contour of lines within the umbra of spots with a maximum intensity, etc.

Investigations of the magnetic field within the sunspot umbra using 'high-temperature' (Fe II, Sr II, Cr II, etc.) and 'low-temperature' (Ti I, Fe I, etc.)** lines may be another point in favour of the inhomogeneous-field model. To make sure that lines Fe II in the sunspot umbra are not associated with the scattered light from the photosphere as claimed by $Z_{\text {waan }}$ (1965), a number of polarization spectrograms in a large

* The systematic understating of the field value on a magnetograph as compared with photographic determinations and which cannot be prevented by calibration may possibly be explained by the effect of averaging the fields $H_{\pi}$ and $H_{\sigma}$ in the magnetosphere.

** 'High-temperature' lines need practically photospheric conditions for excitation, whereas 'lowtemperature' ones, $1500-2000^{\circ} \mathrm{K}$ less. 
E. I. MOGILEVSKY ET AL.
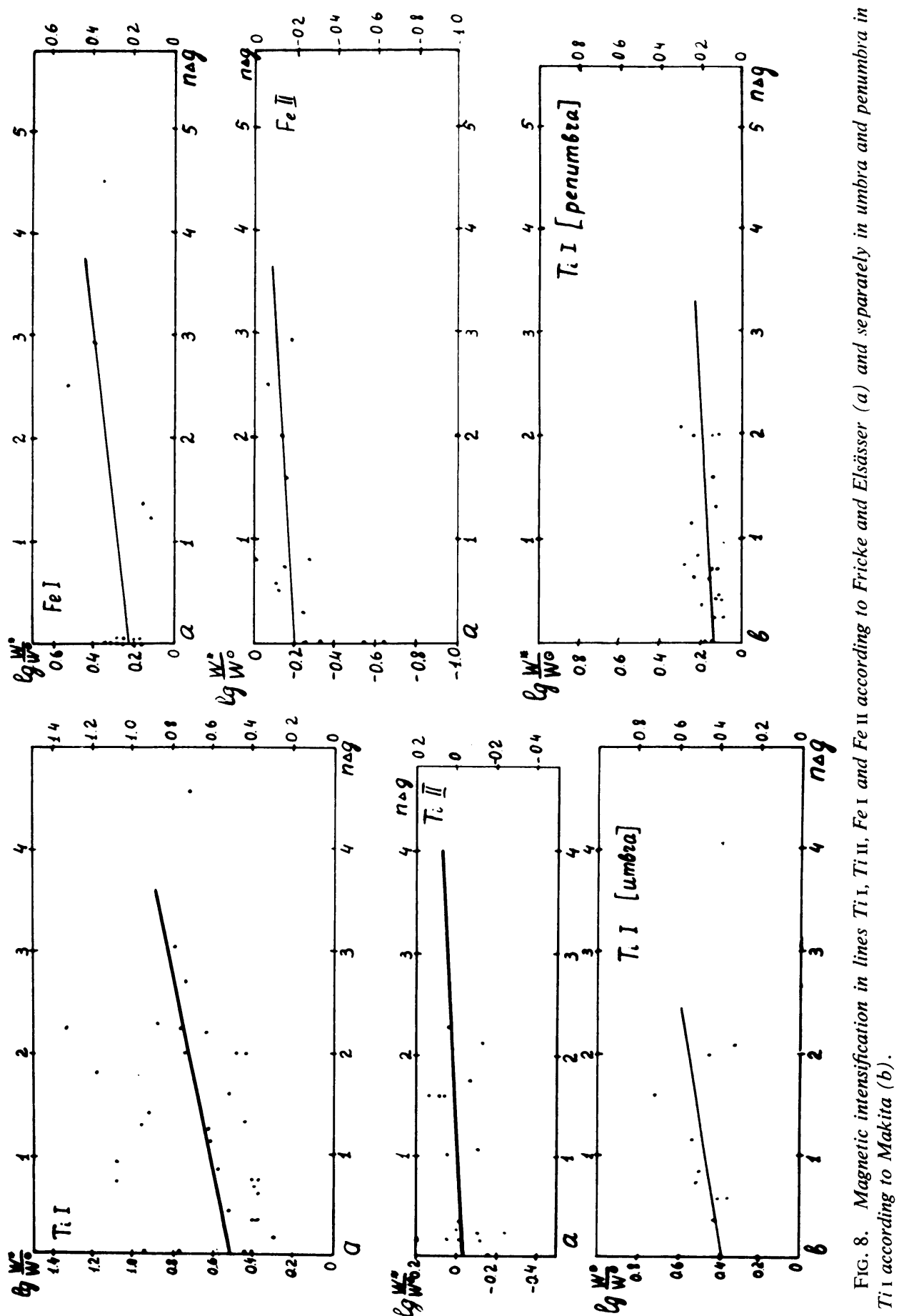
'quiet' spot were obtained with the IZMIRAN solar tower telescope (July-August 1967) in lines Fe II $(\lambda \lambda 5018.45$ and $4924 \AA)$, Fe I $(\lambda 5016.86 \AA)$, and Ti I $(\lambda 5020.03 \AA)$ simultaneously. It was found that the magnetic field in lines $\mathrm{Fe}$ I and $\mathrm{Ti} I$ and $\mathrm{Fe}$ II are similar in the nature of splitting, showing maximum field values at the same points of the sunspot umbra. This indicates that, within the range of possible spatial resolution, lines with different excitation temperatures do coexist in the umbra, and the two-component model of the spot is quite promising (Makita, 1963). Therefore, one could expect that the magnetic fields determined by the lines Ti I and Fe I would be different as well. This question could be considered by investigating the magnetic intensification effect in the sunspot lines. According to Bojarchuk et al. (1960), the logarithm of magnetic intensification $\lg W^{*} / W_{\odot}$ increases linearly with $n \Delta g$, the slope being proportional to the magnetic-field strength. Here $n=$ number of $\sigma$-sub-components, and $\Delta g=$ difference of the Lande factors of the upper and lower levels. Using the data of Fricke and Elsässer (1965) we plotted such a relationship for $\log W^{*} / W_{\odot}$ (where $W$ are equivalent widths) for a number of lines Ti I, Ti II, Fe I, and Fe II.

For the purpose of control, a similar relationship was plotted for the umbra and penumbra for lines $\mathrm{Ti}$ I from the data of Makita (1963). The relatively large scattering of the points is obviously due not only to experimental errors or the difficulty of taking into account the scattered light, but also to the dependence of $\lg W^{*} / W_{\odot}$ on the space orientation of the field, line strength, turbulent velocity, temperature and pressure relations in the umbra and the photosphere. A certain decrease in this scatter was achieved by selecting mean-intensity lines located in the middle part of the growth curve for spots.*

Figure 8 depicts the relationships thus obtained, and Table 1 gives the number of

\section{Table 1}

Fricke and Elsässer 1965

Makita 1963

\begin{tabular}{|c|c|c|c|c|c|c|}
\hline & $\mathrm{Ti}_{\mathrm{I}}$ & Ti II & $\mathrm{Fe}_{\mathrm{I}}$ & $\mathrm{Fe}$ II & Ti I, umbra & Ti I, penumbra \\
\hline & 32 & 20 & 17 & 11 & 11 & 21 \\
\hline & $0.086 \pm 0.045$ & $0.031 \pm 0.027$ & $0.067 \pm 0.012$ & $0.041 \pm 0.032$ & $0.077 \pm 0.035$ & $0.024 \pm 0.020$ \\
\hline & $0.553 \pm 0.077$ & $-0.050 \pm 0.030$ & $0.218 \pm 0.019$ & $-0.210 \pm 0.043$ & $0.395 \pm 0.041$ & $0.144 \pm 0.024$ \\
\hline
\end{tabular}

points used $(N)$ and the coefficients $a$ and $b$ for expressing $\lg W^{*} / W_{\odot}=a n \Delta g+b$, which were obtained by the least-square method. The coefficient $a$ characterizes the magnetic intensification, and is proportional to the magnetic-field strength in the lineformation region, while $b$ defines the increase (or decrease) of the line strength neglecting the magnetic intensification. The intensification in lines $\mathrm{TiI}$ is close to that expected in a field of about 2500-3000 oe (Bojarchuk et al., 1960). In the penumbra

* For such lines magnetic intensification is determined with a high certainty and changes little with small changes in the line strength. 
the intensification is approximately one-third that in the umbra, as could be expected. From the point of view of the present work, a comparison of the magnetic intensifications and the magnetic fields obtained from 'cold' (TiI, FeI) and 'hot' (TiII, FeII) lines, is of special interest. It can be seen from Table 1 that in 'hot' regions the magnetic field is approximately half that in 'cold' ones. Ignoring some weak photospheric lines TiI (with an equivalent width of $\lesssim 10 \mathrm{~m} \AA$ ), which cannot be measured with sufficient confidence, we obtain an even greater magnetic intensification for lines Til, and the field ratio will become about 3.* An analogous relationship (Figure 9) is observed in 9 lines of multiplet No. $38 \mathrm{TiI}(a=0.085, b=0 \cdot 35)$ and, with less certainty, in 5 lines of multiplet No. 49 Fe II $(a=0 \cdot 020, b=-0 \cdot 31)$.

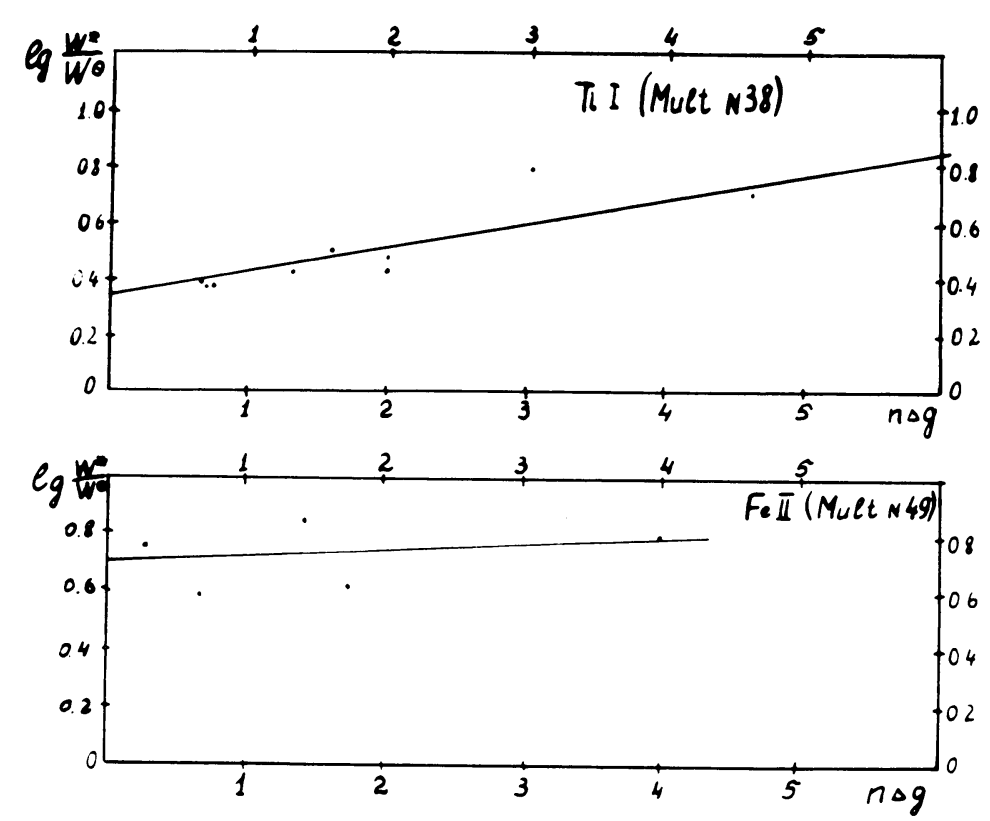

FIG. 9. Magnetic intensification from multiplets $T i \mathrm{I}$ and $F e$ II according to Fricke and Elsässer.

The same difference in the values of the magnetic fields in 'hot' and 'cold' lines was obtained by direct measurements of the magnetic field separately in lines FeII $\lambda \lambda 5018.45 \AA$ and $4923.93 \AA$, FeI $\lambda \lambda 5016.86$ and $4927.78 \AA$, and TiI $\lambda \lambda 5020.03$ and $5016 \cdot 17 \AA$. For this purpose, polarization spectrograms were obtained with the IZMIRAN tower solar telescope for a number of sections in large spots (August 1, 2, and 7,1967$)$.

* An identical ratio is obtained (naturally, with a large scatter) by taking into account all the lines measured in the work (Fricke and Elsässer, 1965), as well as when using data uncorrected for scattered light. 
All these lines were obtained in one spectrogram, thus permitting a comparison of magnetic fields measured from different lines at the same site. Measurements were carried out in 40 independent points of the sunspot umbra and penumbra. Fig. 10a displays the results of these measurements. It also shows the histograms for the occurrence of various values of the ratio $H_{\mathrm{Fe} \mathrm{II}} / H_{\mathrm{Til}}$. Only in 4 of the 40 cases was $H_{\mathrm{Fe} \mathrm{II}} \geqslant H_{\mathrm{Ti}}$, whereas in $70 \%$ of the cases the result was $0 \cdot 4 \leqslant H_{\mathrm{Fe} \mathrm{II}} / H_{\mathrm{TiI}} \leqslant 0 \cdot 6$. The mean

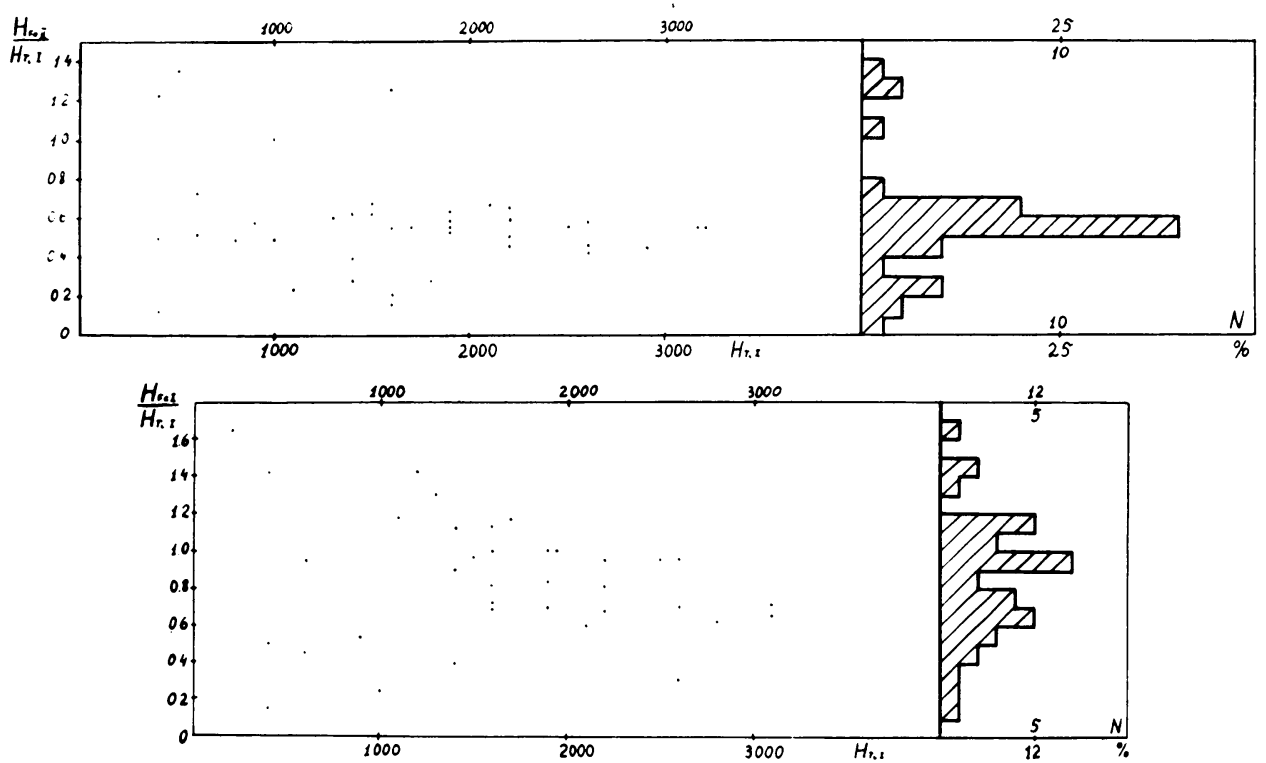

FIG. 10. Variation in magnetic-field ratio from lines $F e \mathrm{II}, T i \mathrm{I}, \mathrm{Fe} \mathrm{I}$ in sunspots of August 1, 2, and $7,1967$.

value of this ratio is 0.55 . Thus, as well as in the case of determining the field from the difference in magnetic intensification, the magnetic field in 'hot' regions is, on the average, half that in 'cold' ones. We may also note that, despite the small number of points used, the ratio $H_{\mathrm{FeII}} / H_{\mathrm{Ti}}$ also exhibits a trend towards the grouping of points around a value of the order of $0 \cdot 25$, which means a four-fold excess of the field of 'cold' regions over that of 'hot' ones. This is close (with some scatter) to the abovementioned values of the magnetic field measured by us from $\pi$ - and $\sigma$-components in line $\mathrm{FeI} \lambda 6302.5 \AA$. The ratios of the magnetic fields measured in the same spectrograms from lines FeI and TiI are presented in Figure 10b. Although in this case the scatter of points is considerable, in $70 \%$ of the cases $H_{\mathrm{Fel}} / H_{\mathrm{Til}} \leqslant 1 \cdot 0$, and the mean value is 0.83 . Here, too, the histogram reveals that the predominant values are $H_{\mathrm{Fel}} / H_{\mathrm{Til}} \approx 1$ and $H_{\mathrm{Fel}} / H_{\mathrm{Til}} \approx 0 \cdot 6$. From all these spectrophotometric data it follows that there is an appreciable inhomogeneity in the values of the magnetic fields, which 
have different values at the same points of the spot (mainly in the umbra), if the field is defined from 'cold' and 'hot' lines.

In the light of the model being considered, this result of spectrophotometric observations points to the existence of a space grouping of sub-granule chains within which temperature conditions are different. Such a difference in temperatures and densities (at equal gas pressure) may arise, for example, due to the oscillatory convection in sub-photospheric layers (Syrovatsky and Zhugzhda, 1967). In such convection cells the sizes and, perhaps, shapes of the subgranules will be different.* Then, even if the total energy of the sub-granule magnetic field is conserved ${ }^{* *}$, the maximum values of the field intensity in the sub-granules of 'hot' cells will be less than those in 'cold' ones. Apparently, in obtaining a spectrogram in the sunspot umbra, a certain space averaging of the effects, introduced by translucent 'hot' and 'cold' cells, takes place. Although not yet sufficiently complete, the accomplished investigations show that in the sunspot field there may exist some small-scale magnetic elements (sub-granules) with a large field and with an intermediate background (small) field of opposite sign between them. The distribution of the sub-granule chains, as well as the geometry of individual sub-granules (i.e. the total energy and maximum intensity of the field), which determine the averaged field observed, is due to the sub-photospheric motions (primarily convection) in the particular site of the sunspot.

\title{
References
}

\author{
Bojarchuk, A. A., Efimov, J.S., Stepanov, V.S. (1960) Astron. J., 37, 812. \\ Bumba, V. (1962) Bull. astr. Inst. Csl., 13, 42. \\ Chistjakov, V.F. (1967) Private communication. \\ Fricke, K., Elsässer, H. (1965) Z. Astrophys., 63, 35. \\ Kadomtzev, B. A. (1962) Nuclear Fusion, 3, 269. \\ Kolpakov, L.E. (1966) Solnechnie dannie, 9, 78. \\ Kuklin, G. V. (1966) The Results of the Observations and Investigations in the Period of the IQSY, \\ $1,16$. \\ Makita, N. (1963) Publ. astron. Soc. Japan, 15, 145. \\ Mogilevsky, E.I., Shelting, B.D. (1966) Proc. of the Meeting on Solar Magnetic Fields and High \\ Resolution Spectroscopy, Firenze, Ed. by G. Barbera, p. 222. \\ Mogilevsky, E.I., Shelting, B.D. (1968) Solar Phys., 4, in press. \\ Neuringer, J.L., Rosenzweig, E.R. (1967) Phys. of Fluids, 7, 1927. \\ Rachkovsky, D.N. (1962) Izv. Crimean Obs., 27, 148. \\ Severny, A.B. (1959) Astr. Zu., 36, 208. \\ Severny, A.B. (1964) Space Sci. Rev., 3, 2. \\ Severny, A. B. (1965) Izv. Crimean Obs., 33, 3. \\ Severny, A. B. (1967) Izv. Crimean Obs., 36, 22.
}

* This follows from the generalized expression of the Bernoulli integral for the sub-granule chain in the outer field $H$. In our case, this relation will have the form $P+\rho v^{2}+\rho g h+x H^{2}=$ const, where $\rho=$ density, $g=$ gravity acceleration, $x=$ effective 'magnetization'.

** At relatively slow deformations the dissipation of the currents flowing inside the sub-granules may occur as well. 
Syrovatsky, S. I., Zhugzhda, Yu.D. (1967) 'The Oscillatory Convection of the Conductive Gas in the Strong Magnetic Fields'. Preprint, No. 15, Phys. Inst. Ac. Sci., U.S.S.R., Moscow. Vassilyeva, G.Y.(1963) Izvestija GAO (Pulkovo), 23, No. 171, 3.

Zhulin, I. A., Ioshpa, B. A., Mogilevsky, E.I., Obridko, V.N. (1966) in Proc. of the Meeting on Solar Magnetic Fields and High Resolution Spectroscopy, Firenze, Ed. by G. Barbera, p. 155.

Zwaan, C. (1965) Recherches astron. Obs. Utrecht, 17. 